\title{
El Real Instituto y Observatorio de la Armada
}

\section{Rafael C. Boloix Carlos-Roca}

Arbor CLXXIII, 682 (Octubre 2002), 349-364 pp.

Desde 1753, en que fue creado por el insigne marino Jorge Juan y Santacilia, el Real Instituto y Observatorio de la Armada (ROA) ha sido el núcleo de la investigación científica de la Armada, manteniendo hoy día un reconocido prestigio tanto a nivel nacional como internacional.

El autor de este artículo, director del ROA, a través de una síntesis descriptiva de la estructura orgánica del centro en cuatro Secciones (Efemérides, Astronomía, Geofisica, y Hora), nos introduce en la evolución histórica de esta institución y en la amplia gama de actividades que desarrolla en la actualidad, participando en numerosos proyectos y foros internacionales, $y$ manteniendo relaciones de colaboración permanente con organismos científicos similares de diversos países.

Sus publicaciones de efemérides astronómicas, sus trabajos en geomagnetismo, sismología, seguimiento de satélites, astrofísica, metrología, etc. etc., así como el hecho de ser el depositario del Patrón Nacional de la unidad de tiempo, son el exponente de que el ROA continúa siendo un centro científico en plena actividad y pujanza, y en no pocas ocasiones la voz de España en el concierto internacional de estas ramas del conocimiento y de la investigación. Finaliza el artículo con una breve referencia a la Escuela de Estudios Superiores, vinculada al ROA desde su creación y que continúa con sus cursos de «estudios mayores» manteniendo vivo el espíritu científico de la Armada, mediante la formación de un selecto núcleo de oficiales en las áreas de ciencias Físico-Matemáticas, Astronomía y Geofisica, y en la preparación para los cursos de Ingeniería. 


\section{Introducción}

Los avances que hoy día hemos alcanzado y disfrutamos se deben al continuo esfuerzo que la humanidad ha dedicado a la ciencia que, como define el Diccionario de la Lengua Española (RAE), es el «conocimiento cierto de las cosas por sus principios y causas». La técnica, por otro lado, no es más que el adecuado empleo y utilización de los procedimientos y conocimientos que la ciencia facilita $\mathrm{y}$, aun siendo muy importante, está subordinada a la ciencia; piénsese que siempre se habla de investigación y desarrollo y no de lo contrario. Es necesario, en primer lugar, el conocimiento cierto de las cosas por sus principios y causas, es decir, la investigación; y luego, con base en estos conocimientos, efectuar su aplicación, el desarrollo.

La Armada, a lo largo de su historia, repetidas veces ha dado prueba de que ha sabido entender y mantener viva esta mentalidad. Cuando lo ha considerado necesario para la adecuada formación, mejora y preparación de sus fuerzas, no ha dudado en potenciar la formación de sus oficiales en el nivel científico superior que estimó pertinente, ni en crear los centros científicos y técnicos que le eran precisos. Algunos de ellos continúan en su seno, otros lo están dentro del ámbito del Ministerio de Defensa, y otros continúan su andadura prestando su servicio al país desligados totalmente del estamento militar, bien como instituciones independientes, bien como facultades o cátedras en las diversas universidades españolas, como es el caso de la cirugía, cuyo inicio en España hay que remontarlo al año 1748 con la aprobación y entrada en funcionamiento, dentro de la Armada, del Real Colegio de Cirugía de Cádiz.

Entre las instituciones de este carácter que continúan perteneciendo a la Armada se encuentra el Real Instituto y Observatorio de la Armada (ROA), centro científico fundado en 1753 a propuesta del insigne marino Jorge Juan y Santacilia, ejemplo vivo de la mentalidad científica de la Armada. A lo largo de su historia, próxima a cumplir doscientos cincuenta años, este centro se ha hecho acreedor de un reconocido prestigio tanto en el ámbito nacional como internacional y por él han pasado prestigiosos marinos, como el propio Jorge Juan, Tofiño, Malaspina, Vargas Ponce, Churruca, Ciscar, Cecilio Pujazón y un largo etc. Los denominados «estudios mayores», que iniciaron en el Observatorio un flujo continuo de formación de oficiales de la Armada, a un nivel superior de conocimientos científicos, continúan materializándose hoy día en su Escuela de Estudios Superiores.

En el Prólogo a la Memoria de las actividades del Observatorio del año 2000, se dice que una visita rápida al Observatorio, en la 


\section{El Real Instituto y Observatorio de la Armada}

que se nos presenta ante la vista un atractivo edificio del siglo XVIII, que alberga una magnífica biblioteca y una amplia colección de instrumentos antiguos, nos puede hacer creer que el Observatorio es un museo, cuando la realidad es bien distinta . Al igual que en ese prólogo, se pretende hacer ver en este artículo que el Observatorio es un centro científico en plena actividad y pujanza en diversas ramas del saber; y para ello, nada mejor que constatar, con un breve recorrido descriptivo, las múltiples actividades que se llevan a cabo en las cuatro secciones en que se estructura el Observatorio (Efemérides, Astronomía, Geofísica, y Hora), para finalizar con unas pinceladas sobre la Escuela de Estudios Superiores y su Biblioteca.

\section{Sección de Efemérides}

La Sección de Efemérides es la más antigua de las cuatro que conforman la estructura básica del Observatorio. Sus orígenes se remontan a 1794, año en que se crea la Oficina de Efemérides, consolidándose formalmente como uno de los pilares del Observatorio con el primer Reglamento, aprobado en 1831.

El Reglamento del Instituto y Observatorio de Marina actualmente en vigor, establece en su artículo 1.1.1. como una de las misiones de este centro «Los estudios teóricos y cálculos relacionados con las efemérides y la publicación de las mismas, realizados dentro de las normas reconocidas internacionalmente y en forma adecuada a sus aplicaciones varias, preferentemente a las náuticas y geodésicas».

Esta misión la ha venido desempeñando la Sección de Efemérides desde su creación; su resultado final ha sido la publicación de las efemérides astronómicas necesarias en navegación, astronomía y geodesia, entendiendo por efemérides astronómicas, de una forma general, la comunicación de datos relativos a fenómenos astronómicos que han de producirse en el futuro, tales como posiciones de los astros, ortos, ocasos, eclipses, etc. El Observatorio inició esta labor en 1791 con la edición del Almanaque Náutico y Efemérides Astronómicas para el año bisiesto de 1792, y desde entonces se ha mantenido sin interrupción.

Los avances científicos y los desarrollos tecnológicos producidos desde la publicación de las primeras efemérides han supuesto la lógica evolución de las efemérides astronómicas actualmente editadas por la Sección.

Para la adecuada realización de esta tarea la Sección entiende de los problemas relacionados con la Mecánica Celeste. Aunque tradicionalmente se venían tratando problemas relativos a los objetos del sistema solar, como parte fundamental del cálculo de las efemérides 
astronómicas, en los últimos años se han abordado, además, problemas de Mecánica Celeste en su vertiente más teórica y en relación con la teoría del satélite artificial, en estrecha colaboración con universidades españolas y extranjeras. La era espacial ha supuesto el resurgir de esta ciencia, recuperando viejas teorías y abriendo un sinfín de nuevos campos de investigación.

Dentro de la gran variedad de problemas abiertos en esta disciplina, en esta Sección se trabaja fundamentalmente en dos líneas distintas: El estudio de la estabilidad de ciertos sistemas dinámicos, y la determinación de las denominadas órbitas periódicas.

La primera de ellas, de carácter esencialmente teórico, trata del estudio de la estabilidad de soluciones de equilibrio de sistemas dinámicos, como es el caso de los satélites geoestacionarios. Si bien el estudio realizado sobre la estabilidad de estos satélites carece de una aplicación práctica inmediata, su conclusión ha llenado el vacío existente en esta parte de la teoría, contribuyendo a la comprensión cualitativa del problema y al progreso de las ciencias matemáticas en general. La segunda línea de trabajo, determinación de órbitas periódicas, es un campo de una mayor aplicación práctica, puesto que el conocimiento de dichas órbitas es muy importante para el diseño de misiones espaciales. Los trabajos realizados en este área, iniciados con miembros de la Universidad de Zaragoza, han supuesto colaboraciones con el «Centre National d'Etudes Spatiales»(CNES), con el Departamento de Ingeniería Aeroespacial de la Universidad de Michigan (para la determinación de zonas seguras alrededor de asteroides) y con la Universidad Politécnica de Madrid (para el estudio del comportamiento de «tethers» o amarras espaciales).

Las publicaciones más relevantes que en este campo edita el Observatorio son las siguientes:

- Almanaque Náutico. Publicación anual que contiene las efemérides del Sol, la Luna, los planetas observables y las estrellas más brillantes, necesarias para resolver los problemas de navegación astronómica. Incorpora, además, un suplemento para la navegación aérea; y desde el año 1997 un Suplemento al Almanaque Náutico, con los ortos, ocasos y crepúsculos del Sol para latitudes extremas.

- Efemérides Astronómicas. También de carácter anual, esta publicación va dirigida fundamentalmente a los astrónomos y a los geodestas. En ella se facilitan efemérides precisas de los objetos celestes y de los principales fenómenos astronómicos du- 
rante el año, así como los datos necesarios para realizar los cálculos que intervienen en la preparación y reducción de las observaciones astronómicas.

\section{Seccion de Astronomía}

A mediados del siglo XVIII la preocupación esencial de los marinos era la de conocer los elementos que les permitieran situarse en alta mar (hallar su latitud y longitud) y fijar su rumbo. La determinación de la latitud, mediante la medida de la altura de la estrella Polar o del Sol a su paso por el meridiano, no representaba una mayor dificultad, pero el conocimiento de la longitud no estaba resuelto de una forma asequible. La resolución de este problema exigía, por un lado, el desarrollo de una relojería más exacta, y por otro, el aumento en la precisión de las tablas que facilitaban la posición de los astros sobre la esfera celeste (efemérides). La fundación del Real Observatorio de Cádiz estaba orientada a mejorar, mediante observaciones astronómicas, la precisión con que se conocían las posiciones de los astros, así como proporcionar al navegante los elementos necesarios para la navegación astronómica.

Fiel a esta tradición, la Sección de Astronomía desarrolla su actividad dentro del campo de la Astronomía de Posición (Astrometría). Su misión fundamental es la determinación de la posición de los astros y otras magnitudes relacionadas con la Astrometría. A lo largo de su existencia no sólo se ha alcanzado, siempre en colaboración con otros Observatorios, la precisión en la posición de los objetos celestes que los navegantes requieren para situarse en la mar, sino que se ha ido más allá, conforme la ciencia ha avanzado y han surgido nuevas exigencias. Una de ellas ha venido de la mano de los vehículos espaciales; en la segunda mitad del siglo XX ha sido la navegación espacial, no la marítima, la que ha solicitado de los observatorios astronómicos la mejora en la determinación de posiciones de los cuerpos celestes.

Los trabajos en este campo los lleva a cabo la Sección de Astronomía con dos instrumentos casi gemelos: el Círculo Meridiano Automático Carlsberg (CAMC) y el Círculo Meridiano de San Fernando (CMASF). Por sus ubicaciones, este centro tiene la capacidad de observar desde $90^{\circ} \mathrm{N}$ a $90^{\circ} \mathrm{S}$.

Desde 1980 el Observatorio de la Armada participa junto a los Observatorios de Greenwich (RGO) y de la Universidad de Copenhague, gracias a la financiación de la Dirección General de Investigación Científica y Técnica, en los trabajos de observación e investigación del Círculo Meridiano danés, instalado en el Observatorio del Roque de 
los Muchachos de la isla de La Palma, y que es conocido como Círculo Meridiano Automático Carlsberg (CAMC).

Este proyecto internacional comprende la observación de estrellas y planetas, análisis de resultados de los instrumentos de observación y la participación en la elaboración y publicación de la serie de catálogos «Carlsberg Meridian Catalogue. La Palma». Estos catálogos, por la calidad de sus datos y por el amplio conjunto de objetos celestes que contienen, constituyen una contribución muy importante a la Astrometría Fundamental, definición de los Sistemas de Referencia Estelares, Dinámica Galáctica, Sistema Solar y estudios de interés astrofísico. La publicación de los resultados obtenidos con el CAMC ha permitido detectar los errores sistemáticos del catálogo FK5 (Fundamental Katalog Nr 5), uno de los más importantes sistemas fundamentales de referencia estelar.

El Círculo Meridiano de San Fernando CMASF fue convenientemente modificado, automatizado y posteriormente trasladado a Argentina en junio de 1996, a la estación de altura Carlos Ulrrico Cesco (CUC), perteneciente al Observatorio Felix Aguilar (OAFA) de la Universidad de San Juan, en la vertiente oriental de los Andes, en latitud $32^{\circ} \mathrm{S}$, longitud $69^{\circ} \mathrm{W}$ y una altitud de 2330 metros.

En octubre de 1997 comenzaron las observaciones válidas para la formación del primer catálogo del CMASF en el hemisferio sur. Los trabajos realizados con este instrumento han dado lugar ya a la publicación de un primer catálogo, con el que se amplía sensiblemente la calidad de las posiciones de las estrellas débiles del hemisferio sur. A partir de enero de 2000 comenzó un nuevo programa de observaciones para la elaboración de un mapa de estrellas hasta la magnitud 16 y declinaciones comprendidas entre $3^{\circ} \mathrm{N}$ y $60^{\circ} \mathrm{S}$, y se están observando del orden de 30000 objetos celestes por noche generando un volumen de información de 2 Gbytes diarios.

Dentro de los trabajos que se desarrollan con las observaciones realizadas con ambos instrumentos se enmarca el Proyecto de Investigación «Formación de Catálogos Astrométricos de $-90^{\circ}$ a $+90^{\circ}$ con los Círculos Meridianos Automáticos Carlsberg en La Palma y el del Real Instituto y Observatorio de la Armada en Argentina. (Segunda fase). (PB97-1437).»

El trabajo realizado mediante observaciones meridianas se ha complementado, en el Observatorio de San Fernando, mediante observaciones astrofotográficas. Esta actividad se ha llevado a cabo con el Astrógrafo Gautier, instrumento que ha prestado un magnífico servicio desde el año 1899 en que se inició el proyecto internacional de «La carta del cielo» y que finalizó en 1929. Fruto de este trabajo fue la 


\section{El Real Instituto y Observatorio de la Armada}

publicación del catálogo correspondiente a la zona asignada a España y que contiene unas 250.000 estrellas. Posteriormente, en colaboración con otros centros se han estudiado las posiciones fotográficas de estrellas, galaxias, planetas, etc. En la actualidad, la actividad astrofotográfica es escasa, debido fundamentalmente a la deficiente calidad de cielo en la zona de San Fernando causada por la polución.

La ciencia moderna exige conocer con todo detalle cómo gira la Tierra, no sólo para satisfacer la necesidad científica en sí de obtener el conocimiento más profundo sobre el comportamiento del cuerpo que nos soporta, sino por las consecuencias prácticas que de este conocimiento se derivan para su aplicación en la navegación espacial, el establecimiento de escalas de tiempo astronómico, la Radioastronomía, y la posibilidad de seguir los efectos precursores o posteriores a los grandes seísmos. Desde 1968 hasta 1983, el Observatorio de la Armada, con el Astrolabio Danjon, participó de forma activa en este campo de trabajo y es de destacar que fue el único en España que colaboró en la determinación de la Hora astronómica, a escala global. Si bien es cierto que los avances alcanzados por otras técnicas, fundamentalmente la utilización de las constelaciones de satélites GPS, han hecho obsoleto a este instrumento en este tipo de trabajo, en la actualidad, tras su adecuada transformación, su actividad se centra en el estudio de las medidas del diámetro del Sol, lo que constituye una aportación especialmente apreciada por los astrofísicos, que ven en estas medidas una manera de confirmar sus teorías sobre los fenómenos de física solar que implican unas determinadas variaciones de su diámetro, así como la respuesta de la atmósfera terrestre a la actividad solar.

\section{Sección de Geofísica}

Se entiende por Geofísica la ciencia que estudia los aspectos físico-matemáticos de los fenómenos relacionados con la Tierra. Tradicionalmente se divide en siete grandes campos: Geodesia, Sismología, Meteorología, Geomagnetismo, Física de los Océanos, Vulcanología e Hidrología. De todas estas disciplinas, en la Sección de Geofísica del Observatorio se trabaja de forma asidua en geomagnetismo, sismología, geodesia y meteorología, radicando además en ella el Observatorio Geofísico de la Armada. La Sección se estructura en tres Servicios principales: Sismología, Geomagnetismo y Satélites, además de las actividades y trabajos relacionados con la Meteorología. 


\section{Servicio de Geomagnetismo}

Nace en 1879 con la finalidad de estudiar el campo geomagnético y controlar la variación de la referencia geomagnética, por su directa aplicación en la cartografía náutica. En dicho año se instala en el recinto del Observatorio de la Armada una estación variométrica (relativa) de tres componentes, modelo Adie, complementada por un magnetómetro Elliot y un teodolito Dower, para la observación de las referencias absolutas. Con su instalación nace el primer observatorio geomagnético de España, cuya serie temporal de datos es la tercera más larga del mundo. En la actualidad cuenta con una instalación con la que se efectúan: Medidas relativas de las tres componentes del campo magnético (variación en el tiempo), mediante diversas estaciones variométricas, y medidas absolutas del campo geomagnético (bases de referencia), con diversos magnetómetros e inclinómetro-declinómetros.

Desde septiembre de 1996 la estación magnética de este centro forma parte de la Red Global de Observatorios Geomagnéticos, designada como INTERMAGNET, y colabora de forma regular con el «World Data Centre» (WDC, Boulder, USA), y el «British Geological Survey» (BGS, Edimburgo, Reino Unido), etc. Asimismo, los datos observacionales obtenidos contribuyen a configurar el Campo Geomagnético Internacional de Referencia (IGRF), publicado por la Asociación Internacional de Geodesia y Aeronomía (IAGA) a partir de datos de los observatorios terrestres y de satélites artificiales, y que son imprescindibles para calibrar y controlar los sensores geomagnéticos embarcados en satélites artificiales (CHAMP, Oersted, etc.), proyectos internacionales con los que también se colabora.

Además de la labor propia de observatorio geomagnético, otra de las líneas de trabajo del Servicio de Geomagnetismo es la participaciór. en campañas marinas. En esta línea se pueden mencionar las campañas anuales de la Zona Económica Exclusiva Española (ZEEE), en las que participa este Observatorio desde el año 1996, y en las que mediant el empleo como plataforma marina del BIO Hespérides, durante un me: al año, se está procediendo al levantamiento sistemático de la citadi ZEEE por medio de diferentes técnicas geofísicas. Como aplicaciones pu ramente científicas, podrían citarse un gran número de campañas marina realizadas en los últimos años en colaboración con diversas institucione y organismos internacionales; baste mencionar que en el año 2000, : sólo en el Golfo de Cádiz, se ha participado en tres de dichas campañas designadas como Parsifal, Tasyo y Anastasia, financiadas tanto me diante fondos nacionales como de la Unión Europea. 


\section{El Real Instituto y Observatorio de la Armada}

Finalmente, cabe mencionar como un último tipo de actividades, el apoyo en la calibración de equipos geomagnéticos que con carácter esporádico presta este Servicio.

\section{Servicio de Sismología}

Se crea en 1898 con la finalidad de participar en el estudio de la sismicidad a nivel global. En dicho año se instala en el recinto del Observatorio el sismógrafo horizontal número 6 de Milne, primer sismógrafo instalado en España y uno de los 12 sismógrafos de la primera red sísmica desplegada a nivel mundial. Con su instalación nace la sismología instrumental en nuestro país.

La posición geográfica del Observatorio, próximo a la frontera entre las placas continentales Eurasiática y Africana, le permite disfrutar de un lugar privilegiado para el estudio de la sismicidad de esta región, con las consiguientes aplicaciones que dicho estudio tiene en la predicción y prevención sísmicas. Para dicho fin se dispone en la actualidad de la siguiente instrumentación:

- Red Sísmica de Corto Período: Consta en la actualidad de 10 estaciones sísmicas de corto período. Hasta la actualidad se han detectado más de 5000 terremotos en la zona desde la fecha de instalación de la red.

- Estación Sísmica de Largo Período: Consta de 3 sismómetros Sprenghether instalados desde 1978 en los túneles del Observatorio, y está orientada a la detección y registro de la sismicidad lejana.

- Red de Estaciones de Banda Ancha: Formada por un conjunto de estaciones sísmicas de banda ancha instaladas en las proximidades del Puerto de Santa María y Jerez (1996), Estación Naval de la Algameca (Cartagena, 1997), Estación Naval de Mahón (Menorca, 1998) y Ciudad Autónoma de Melilla (1999). Estas estaciones se encuentran asociadas a la red mundial GEOFON y, a través de ésta, a otras redes mundiales tales como ORFEUS, IRIS, etc. Asimismo, todas estas estaciones participan en la actualidad en el proyecto europeo MIDSEA, coordinado por el Instituto ETH de Zurich (Suiza).

$\mathrm{Al}$ igual que sucedía con el Servicio de Geomagnetismo, el Servicio de Sismología desarrolla diferentes actividades de investigación y técnicas, además de la labor propia como observatorio. En esta línea, participa de forma asidua en campañas de Geofísica de Refracción/Reflexión Sísmica, mediante el empleo de fuentes de energía en la mar 
o en tierra (p.e. cañones de aire del BIO Hespérides o explosiones controladas en la mar) y registro en estaciones sísmicas portátiles desplegadas en tierra a lo largo de perfiles predefinidos, o mediante hidrófonos remolcados en la mar. Este tipo de campañas está orientado al estudio de la corteza y manto superior terrestre (hasta una profundidad de $40-50 \mathrm{~km}$.), y podrían citarse como ejemplos más significativos las ya mencionadas campañas Tasyo y Parsifal, desarrolladas en aguas del Golfo de Cádiz, o la campaña GEODEC-GEOMAR desarrollada en aguas del Mar de Brandsfield (Antártida) en los meses de enero y febrero de este año.

La difusión de los resultados de los trabajos en este campo se realiza a través de los Boletines Provisionales de Sismos y Anales de la Sección de Geofísica, y con la presentación de trabajos en foros nacionales e internacionales, tales como la Asamblea Hispano-Portuguesa de Geodesia y Geofísica, la Comisión Sismológica Europea (CSE), la Sociedad Geofísica Europea (EGS), la Unión Internacional de Geodesia y Geofísica (IUGG), etc., así como con su publicación en diversas revistas científicas.

\section{Servicio de Satélites}

En febrero del año 1958, y merced a una colaboración con la «Smithsonian Institution» (USA), se instala en el recinto del Observatorio una cámara Baker Nunn, primer método global de seguimiento de satélites artificiales, incorporándose este Observatorio, como primera institución española y europea, a esta nueva disciplina. Con ella, este Observatorio, participó activamente en los 25 primeros años de la era espacial.

A finales de los 60, mediante colaboración con el CNES-CERGA francés, se instala una primitiva estación láser de seguimiento de satélites artificiales, basada en un láser de rubí, antecesora de la actual estación de telemetría láser de segunda generación. La Estación Láser del Observatorio de San Fernando, código internacional 7824, es la única estación láser de seguimiento de satélites de la Península Ibérica y Norte de África que se encuentra integrada en la red europea EUROLAS de estaciones láser, y es miembro del Servicio Internacional de Estaciones Láser (ILRS). En la actualidad, esta estación efectúa el seguimiento de forma sistemática de satélites bajos y medios (6000 $\mathrm{km}$.), tanto en períodos nocturnos como diurnos y con una precisión que está por debajo de los $3 \mathrm{~cm}$. Este hecho ha permitido que en el año 1997, y por primera vez, esta estación láser fuera incluida dentro del grupo de estaciones que participan en la definición del Sistema 


\section{El Real Instituto y Observatorio de la Armada}

Terrestre de Referencia Internacional (ITRF), generado por la Asociación Internacional de Geodesia (IAG), y se está abordando ya la siguiente fase de desarrollo que conducirá al seguimiento de satélites altos $(30.000 \mathrm{~km}$.).

Conviene destacar que el seguimiento láser de satélites artificiales es una de las cuatro técnicas fundamentales en geodesia, junto con la Interferometría de Larga Base (VLBI), el Sistema de Posicionamiento Global (GPS) y el Sistema DORIS (Doppler Orbitography and Radiopositioning Integrated by Satellite). No obstante, la técnica láser de seguimiento de satélites, integrada por tan sólo unas 40 estaciones a nivel mundial, es de importancia fundamental por ser el único procedimiento para medir la variación temporal del campo gravitatorio terrestre, y definir un sistema de referencia geocéntrico con precisión del orden de milímetros; además, es el único sistema capaz de monitorizar el movimiento vertical en un sistema de referencia absoluto, y de detectar redistribuciones de masa de gran escala en el interior de la Tierra, océanos y atmósfera.

Desde el nacimiento del GPS como técnica de aplicación civil en geodesia, a principios de los 80 , el Observatorio incorporó esta actividad dentro de su Servicio de Satélites, mediante la adquisición y puesta en funcionamiento de equipos geodésicos de observación GPS, primeros equipos en España de dichas características. Desde entonces, el Observatorio ha participado en diversas campañas, tales como las Campañas Geodésicas Antárticas, Campaña de Referenciación Europea EUREF'89, EPOCH'92, Campaña de Referenciación de la Península Ibérica IBERIA'95, etc.

Desde dichas fechas y hasta la actualidad el Observatorio ha desplegado diversas redes de observación GPS, de las que cabe destacar las siguientes: Estación permanente de Referencia (1995), que desde el año 1996 está incluida dentro de la red global de estaciones fundamentales IGS (International GPS Service for Geodynamics), coordinada por la Asociación Internacional de Geodesia (IAG); y la Red GPS permanente, formada por cuatro Estaciones Geodésicas GPS permanentes, adicionales a la de San Fernando, instaladas en Cartagena (1997), Mahón (1998), Ciudad Autónoma de Melilla (1998) y Granada (2001).

Todas estas actividades, que se están realizando en el campo de las aplicaciones geodésicas del GPS, tienen una contínua repercusión en el ámbito científico nacional e internacional. Se mantienen colaboraciones permanentes con el Instituto Geográfico Nacional (IGN), Instituto Cartográfico de Cataluña (ICC), U. Complutense de Madrid, U. Politécnica de Madrid, U. Politécnica de Cataluña, U. de Jaén, U. de Cádiz, etc,. Y a nivel internacional existen relaciones con la Red 
Global IGS, Red Europea EUREF, Proyecto Internacional WEGENER, Agencia Espacial Italiana (ASI), CNRS-Cote de Azur (Francia), Danish Meteorological Institute (Dinamarca), Bundestag fur Kartographie et Geodasie (BKG, Alemania), etc.

Además, la difusión de los resultados en este campo se realiza mediante la remisión de artículos a revistas especializadas y con la presentación de trabajos en las Asambleas Generales de la Asociación Internacional de Geodesia (IAG), del proyecto WEGENER, del IGS, de la Unión Internacional de Geodesia y Geofísica (IUGG), en la Asamblea Hispano-Portuguesa de Geodesia y Geofísica (AHPGG), etc.

\section{Meteorología}

A medida que se fue desarrollando la astronomía en el Real Instituto y Observatorio de la Armada, surgió en paralelo la necesidad de tomar datos meteorológicos, con la finalidad de aplicar correcciones a las medidas astrométricas mediante modelos de refracción atmosférica.

Aunque las medidas pluviométricas se remontan en su registro a 1811, los datos meteorológicos no se empezaron a publicar de forma secuencial e ininterrumpida hasta la aparición de los "Anales del Instituto y Observatorio de Marina de San Fernando» en 1870, siendo la serie meteorológica de San Fernando la más extensa de toda España a nivel temporal, y una de las más extensas a nivel global. Asimismo, este Observatorio fue el encargado de organizar y gestionar en su primera época el llamado Servicio Meteorológico Costero, que con el tiempo derivaría en el actual Instituto Nacional de Meteorología.

Desde febrero de 1993, continuando la tradicional colaboración mantenida con el Instituto Nacional de Meteorología (INM), se encuentra instalada en el recinto del Observatorio una Estación Automática Digital Modelo SEAC, integrada en la Red Automática Nacional.

\section{Sección de Hora}

La Sección de Hora del Real Instituto y Observatorio de la Armada en San Fernando se establece como tal en 1972, tras haber estado hasta entonces incluida como Servicio de Hora en la Sección de Astronomía. Está íntimamente ligada, desde sus orígenes, con la determinación y conservación con el máximo rigor y exactitud posibles de las escalas de tiempo astronómico, en función de las sucesivas definiciones de la unidad de tiempo del Sistema Internacional: El segundo. 


\section{El Real Instituto y Observatorio de la Armada}

Tradicionalmente la Armada Española, en su interés por poder determinar con la máxima precisión la posición de sus buques, ha jugado un papel fundamental en el estudio y conservación de las escalas de tiempo, en el entendimiento de que un mejor conocimiento y mayor exactitud de la escala de tiempo conlleva una mayor precisión en la navegación.

Los avances en la determinación de la medida del tiempo por métodos físicos dan paso, tras el nacimiento de los Patrones Atómicos de Cesio, a la definición del Segundo Internacional a partir de una propiedad física del átomo de cesio. Coincidente con el establecimiento de la Escala de Tiempo Atómico Internacional, el entonces Servicio de Hora se transforma en la Sección de más reciente creación del Observatorio.

Por ello, en su origen, no puede desligarse de los trabajos realizados en el seno del Comité Consultivo para la Definición del Segundo, enmarcado en el desaparecido Buró Internacional de la Hora, cuyas funciones asume hoy en día el Buró Internacional de Pesas y Medidas.

Para el desarrollo de sus funciones principales, la Sección está estructurada en tres Servicios: Hora, Cronometría Naval, y Electrónica y Calibración.

\section{Servicio de Hora}

Tiene como misión principal el mantenimiento de la unidad básica de tiempo, declarado a efectos legales como Patrón Nacional de dicha unidad, así como el mantenimiento y difusión oficial de la escala «Tiempo Universal Coordinado» (UTC(ROA)), considerada a todos los efectos como la base de la hora legal en todo el territorio nacional (Real Decreto 23 Octubre 1992, Núm. 1308/1992).

$\mathrm{Su}$ laboratorio está declarado como «asociado al Centro Español de Metrología” (Real Decreto 23 Octubre 1992, Núm. 1308/1992).

Para llevar a cabo el mantenimiento del patrón nacional y de la escala de tiempo UTC(ROA), dispone de una batería de patrones de frecuencia de haz de cesio e instrumentación para adquisición automática y control de datos generados por los patrones propios, así como de la instrumentación auxiliar adecuada. Esta escala, a la que genéricamente se hace referencia en este ambiente de trabajo como UTC(ROA), es decir, Tiempo Universal Coordinado del Real Instituto y Observatorio de la Armada, es la base, como ya se ha mencionado, de la Hora Legal Española. Está considerada por diversos autores extranjeros como una de las diez escalas más estables del mundo. Esta escala de tiempo está trazada a la escala de tiempo atómico 
internacional, TAI, mediante la aportación mensual de las lecturas de los patrones de cesio y la participación de este centro en una serie de intercomparaciones de tiempo utilizando la constelación GPS, y por medio de la técnica más precisa de intercomparación de escalas de tiempo existente en la actualidad: transferencia de tiempo y frecuencia mediante la técnica TWSTFT (Two Way Satellite Time and Frequency Transfer). Los niveles de precisión alcanzados con esta última técnica señalada son tales que, después de un ajuste cuadrático a las medidas, los valores RMS están por debajo de un nanosegundo.

Como institución generadora del patrón nacional de tiempo y frecuencia, el Observatorio representa a España en distintos foros metrológicos internacionales. Así, es el representante español en el Comité Consultivo de Tiempo y Frecuencia del BIPM, actuando como miembro muy activo de distintos grupos de trabajo. Igualmente representa a España en el área de tiempo y frecuencia de EUROMET, organización europea para el intercambio de experiencia e infraestructura metrológica; y un miembro del Observatorio forma parte del Comité Científico del Foro Europeo de Tiempo y Frecuencia, el más importante foro científico europeo en este área.

La difusión de la hora legal se efectúa siguiendo diferentes procedimientos:

- Transmisión de señales horarias en HF, durante dos períodos de 25 minutos diarios, en 15006 y $4998 \mathrm{KHz}$, respectivamente, entre 10:00 y 11:00 horas UTC.

- A través de INTERNET mediante el Protocolo NTP y servidor Hora.roa.es / ntp.roa.es.

- Mediante calibraciones de patrones de tiempo y frecuencia.

- Por medio de las intercomparaciones de medidas que esporádicamente son organizadas por la Entidad Nacional de Acreditación, y que pilota el Laboratorio de Hora del Observatorio.

\section{Servicio de Cronometría Naval}

Es el responsable de toda la cronometría de la Armada, llevando a cabo las labores propias de mantenimiento, reparación y desarrollo de nuevas instrumentaciones.

\section{Servicio de Electrónica y Calibración}

Es el responsable del mantenimiento de los patrones y electrónica asociada, y lleva a cabo la calibración de los diversos patrones de 


\section{El Real Instituto y Observatorio de la Armada}

tiempo que existen en España, tanto en la industria nacional como en el ámbito de la Defensa.

$* * * * * * * * * * * * * * * * * * * *$

Además de las múltiples actividades que desarrollan las cuatro Secciones hasta ahora descritas, el Observatorio tiene encomendada también la importante labor de formar científicamente a algunos oficiales navales en estas ramas del saber, y ser el depositario y mantenedor del espíritu científico que siempre ha existido en la Armada. Para ello, cuenta con una Escuela y una Biblioteca, a las que obligadamente hay que hacer referencia, aunque sea muy brevemente.

\section{La Escuela de Estudios Superiores}

Junto a las actividades científicas, en el Observatorio se lleva a cabo una importante actividad docente, cuyos antecedentes se remontan a la propia fundación del Observatorio y de la Academia de Guardias Marinas, y a la responsabilidad asignada desde su creación de impartir los cursos de «estudios mayores».

Por esta Escuela, cuyos profesores son los Jefes de Sección y Servicio del Observatorio, han pasado un gran número de oficiales que han recibido la formación y preparación que, en cada momento, ha determinado como necesaria la Armada.

En la actualidad, los cursos que en ella se imparten son los siguientes:

- Estudios Superiores en Ciencias Físico-Matemáticas, curso conocido cariñosamente en el seno de la Armada como «curso de sabios».

- Estudios Superiores en Astronomía y Geofísica.

- Estudios complementarios.

- Cursos para el acceso a Ingenieros Navales, Ingenieros de Armas Navales e Ingenieros Hidrógrafos.

\section{Biblioteca}

La creación en 1753 del Real Observatorio de Cádiz, producto de la política ilustrada de la época, fue un hito relevante en el desarrollo científico de la España del siglo XVIII. La conjunción entre enseñanza, práctica e investigación, contribuyó rápidamente al aumento de importancia de su Biblioteca, que desde un primer momento fue considerada como un instrumento científico más. Primero Jorge Juan, y 
más adelante Tofiño, hicieron todo lo posible por consolidar y aumentar sus fondos, de tal forma que, cuando en 1798 el Observatorio de Marina fue trasladado a su nuevo emplazamiento en la Isla de León, en el edificio construido por el Marqués de Ureña ya se reservó una sala para la ubicación de la Biblioteca.

Durante el siglo XIX se produjo un continuo incremento de los fondos bibliográficos del Observatorio, motivado esencialmente por tres causas: la recogida de obras de otros centros de la Armada, la adquisición de libros en el extranjero, y el intercambio de publicaciones con otras instituciones. A partir de 1856, la creación de un Curso de Estudios Superiores con sede en el Observatorio, siguiendo la tradición iniciada en el siglo anterior cuando determinados oficiales de la Armada llevaban a cabo los llamados «estudios mayores», fue origen de la incorporación de un importante número de libros de carácter especializado para las nuevas necesidades docentes.

A lo largo del siglo XX la expansión de la Biblioteca ha continuado a buen ritmo, hasta el punto de que sus dependencias ocupan actualmente la mayor parte del edificio principal del Observatorio. Sus más de 30.000 volúmenes, incluida la importante colección de publicaciones periódicas, forman una de las más interesantes bibliotecas científicas del país y constituyen un elemento inseparable del quehacer científico del Observatorio y de las tareas docentes de la Escuela de Estudios Superiores de la Armada. Junto a la magnifica colección de libros, la Biblioteca cuenta con una interesante colección de material cartográfico (más de 3.000 mapas), compuesta principalmente por cartografía náutica de las costas españolas de los siglos XVIII y XIX.

Además, actualmente la Biblioteca tiene a su cargo el Archivo Histórico y la Colección de Instrumentos Antiguos, es decir, el patrimonio histórico tanto documental como científico de la institución.

$* * * * * * * * * * * * * * * *$

No pueden finalizar estas líneas sin reconocer expresamente que el prestigio que el Real Instituto y Observatorio de la Armada posee en el ámbito científico nacional e internacional, y que redunda en el de la propia Armada y en el de España, se debe al esfuerzo, trabajo y estudio continuado de todos y cada uno de los que, a lo largo de su historia, han trabajado y trabajan en él. 